\title{
Video Article \\ Polydimethylsiloxane-polycarbonate Microfluidic Devices for Cell Migration Studies Under Perpendicular Chemical and Oxygen Gradients
}

\author{
Hung-Ju Chiang ${ }^{1}$, Sih-Ling Yeh ${ }^{1}$, Chien-Chung Peng ${ }^{1}$, Wei-Hao Liao ${ }^{1}$, Yi-Chung Tung \\ ${ }^{1}$ Research Center for Applied Sciences, Academia Sinica
}

Correspondence to: Yi-Chung Tung at tungy@gate.sinica.edu.tw

URL: https://www.jove.com/video/55292

DOI: doi:10.3791/55292

Keywords: Bioengineering, Issue 120, Polydimethylsiloxane, Polycarbonate, Microfluidics, Chemical Gradient, Oxygen Gradient, Cell Culture, Cell Migration

Date Published: 2/23/2017

Citation: Chiang, H.J., Yeh, S.L., Peng, C.C., Liao, W.H., Tung, Y.C. Polydimethylsiloxane-polycarbonate Microfluidic Devices for Cell Migration Studies Under Perpendicular Chemical and Oxygen Gradients. J. Vis. Exp. (120), e55292, doi:10.3791/55292 (2017).

\section{Abstract}

This paper reports a microfluidic device made of polydimethylsiloxane (PDMS) with an embedded polycarbonate (PC) thin film to study cell migration under combinations of chemical and oxygen gradients. Both chemical and oxygen gradients can greatly affect cell migration in vivo; however, due to technical limitations, very little research has been performed to investigate their effects in vitro. The device developed in this research takes advantage of a series of serpentine-shaped channels to generate the desired chemical gradients and exploits a spatially confined chemical reaction method for oxygen gradient generation. The directions of the chemical and oxygen gradients are perpendicular to each other to enable straightforward migration result interpretation. In order to efficiently generate the oxygen gradients with minimal chemical consumption, the embedded PC thin film is utilized as a gas diffusion barrier. The developed microfluidic device can be actuated by syringe pumps and placed into a conventional cell incubator during cell migration experiments to allow for setup simplification and optimized cell culture conditions. In cell experiments, we used the device to study migrations of adenocarcinomic human alveolar basal epithelial cells, A549, under combinations of chemokine (stromal cell-derived factor, SDF-1 $\alpha$ ) and oxygen gradients. The experimental results show that the device can stably generate perpendicular chemokine and oxygen gradients and is compatible with cells. The migration study results indicate that oxygen gradients may play an essential role in guiding cell migration, and cellular behavior under combinations of gradients cannot be predicted from those under single gradients. The device provides a powerful and practical tool for researchers to study interactions between chemical and oxygen gradients in cell culture, which can promote better cell migration studies in more in vivo-like microenvironments.

\section{Video Link}

The video component of this article can be found at https://www.jove.com/video/55292/

\section{Introduction}

The spatial distribution of soluble factors and oxygen tension can regulate a number of important cellular functions in vivo ${ }^{1,2,3,4}$. In order to better investigate their effect on cells, an in vitro cell culture platform capable of stably generating chemical and oxygen gradients is highly desired. Various soluble factors play key roles in biological activities and affect cellular behavior. Recently, due to the advancement of microfluidic techniques, a number of microfluidic devices capable of stably generating chemical gradients have been developed to study cell migration ${ }^{5}$. Furthermore, several studies have also revealed the necessity of oxygen tension for in vitro cell cultures ${ }^{6,7,8}$. However, the control of oxygen tension for cell culture mainly relies on direct chemical addition for oxygen scavenging or cell incubators with pressurized gas cylinders. Direct chemical addition alters the cell culture medium and affects the cellular responses. Oxygen control incubators require special incubator design, precise gas flow control, and a large volume of nitrogen gas in order to achieve hypoxia conditions. Moreover, it is infeasible to control the spatial distribution of oxygen using this setup, which retards the cellular behavior study under various oxygen tensions and gradients. To overcome these limitations, a number of microfluidic devices have been developed to generate oxygen gradients for cell culture applications ${ }^{9}$. However, most of them are operated using pressurized gases, which may cause evaporation and bubble generation problems. Therefore, they often require sophisticated instrumentation and may not be reliable for long-term cell culture studies.

To overcome the challenges and to further study the interactions between chemical and oxygen gradients for cell migration, we developed a microfluidic cell culture device made of polydimethylsiloxane (PDMS) with an embedded polycarbonate (PC) thin film ${ }^{10}$. The device is composed of two microfluidic channel layers separated by a PDMS membrane. The top layer is a PDMS-PC layer for oxygen gradient generation; the bottom layer is made of PDMS for chemical gradient generation and cell culture. The device can simultaneously generate perpendicular chemical and oxygen gradients without using gas cylinders and sophisticated flow control systems. In the device, PDMS provides great optical transparency, gas permeability, and biological compatibility for cell culture and imaging. The embedded PC film serves as a gas diffusion barrier for efficient oxygen tension control. In the microfluidic channel, we used a series of serpentine-shaped channels to generate chemical gradients. The design has been broadly exploited to generate chemical gradients in microfluidic devices for various applications due to its reliability and easy experimental setup. Furthermore, the chemical gradient profiles can be designed by varying the channel geometries beforehand with numerical simulation. For oxygen gradient generation, we took advantage of the spatially confined chemical reaction method that was previously developed in our lab ${ }^{10,11,12}$. The oxygen can be scavenged from the designated areas without nitrogen purging. For practical usage in biological 
labs, the entire experimental setup is compatible with conventional cell culture incubators. By integrating these methods, we can simultaneously generate stable chemical and oxygen gradients without bulk gas cylinders and sophisticated instrumentation in order to study cell migration.

\section{Fabrication of the Microfluidic Device}

NOTE: The entire microfluidic device is fabricated using the soft lithography replica molding process ${ }^{13}$.

\section{Fabrication of molds for the PDMS layers in the microfluidic device}

1. Design the microfluidic channel patterns using commercially available illustration or drawing software. Submit the file to a company for high-resolution transparency photomask printing ${ }^{14}$.

2. Clean silicon wafers with acetone ( $\geq 99.5 \%$ ), isopropyl alcohol (IPA; $\geq 99.9 \%)$, and buffered oxide etch (BOE; $\left.6: 1 \mathrm{NH}{ }_{4} \mathrm{~F} . \mathrm{HF}\right)$. Rinse with deionized (DI) water and dehydrate the wafer with a nitrogen gun.

3. Spin coat approximate $20 \mathrm{~g}$ of negative tone photoresist, SU-8 2050, onto the wafers at $500 \mathrm{rpm}$ for $15 \mathrm{sec}$ and then $2,000 \mathrm{rpm}$ for 30 sec.

NOTE: The spin coating condition should yield SU-8 2050 photoresist layers with a thickness of approximately $75 \mu \mathrm{m}$ on the wafers after optical lithography.

4. Soft bake the wafers on a $65^{\circ} \mathrm{C}$ hotplate for $3 \mathrm{~min}$ and then at $95^{\circ} \mathrm{C}$ for $9 \mathrm{~min}$. After the soft bake, expose the wafers using a mask aligner with the designed transparency masks under UV light; the total exposure energy should be about $300 \mathrm{~mJ} / \mathrm{cm}^{2}$. Post-exposure bake (PEB) the wafers at $65^{\circ} \mathrm{C}$ for 2 min and then at $95^{\circ} \mathrm{C}$ for $7 \mathrm{~min}$.

5. After the PEB, immerse the wafers in SU-8 developer with strong agitation or in an ultrasonic bath ( $37 \mathrm{kHz}$ and $180 \mathrm{~W}$ effective ultrasonic power) for $7 \mathrm{~min}$. Rinse the wafer with acetone and IPA to remove the residual SU-8.

6. Place the wafer and $100 \mu \mathrm{l}$ of silane $(1 \mathrm{H}, 1 \mathrm{H}, 2 \mathrm{H}, 2 \mathrm{H}$-perfluorooctyltrichlorosilane) into a $6 \mathrm{~cm}$ diameter Petri dish in a desiccator connected with a diaphragm vacuum pump for wafer surface silanization in order to prevent undesired bonding. Turn on the pump for $15 \mathrm{~min}$, turn it off, and seal the desiccator with a vacuum for $30 \mathrm{~min}$.

7. Take the silanized wafers out of the desiccator and tape them to $15 \mathrm{~cm}$ diameter Petri dishes for the following soft lithography process:

\section{Fabrication and assembly of PDMS layers}

1. Preparation of the PDMS pre-polymer

1. Mix PDMS monomer (base) and the curing agent at a 10:1 ratio (v/v). Degas the mixture in the desiccator setup for 60 min.

2. Fabrication of the PC-embedded top layer

1. Transfer $2 \mathrm{~g}$ of the PDMS pre-polymer onto the mold with the top-layer fluidic channel patterns to make a thin layer of PDMS. Place the mold in the desiccator setup to degas the PDMS for 60 min.

2. Place the mold overnight in a $60^{\circ} \mathrm{C}$ oven for PDMS curing. Make sure that the mold is on a horizontal plane.

3. Cool down the mold to room temperature. Pour an additional $13 \mathrm{~g}$ of PDMS pre-polymer onto the mold and degas the PDMS in the desiccator setup for $60 \mathrm{~min}$.

4. Slowly embed a $1 \mathrm{~mm}$ thick PC film into the fresh PDMS layer as a gas diffusion barrier; expel any bubbles if needed.

5. Put the mold overnight in the $60^{\circ} \mathrm{C}$ oven, and make sure that it is on a horizontal plane.

6. Cool down the cured PDMS to room temperature. Cut the device with a scalpel to an area of approximately $5.5 \times 5 \mathrm{~cm}^{2}$, which can cover all channel patterns, and peel off the PDMS slab from the mold.

7. Punch holes for inlets and outlets using a $2 \mathrm{~mm}$-diameter biopsy punch. Store the fabricated top PDMS-PC layer away from ambient dust for later assembly.

3. Fabrication of the bottom PDMS layer

1. Pour $11 \mathrm{~g}$ of the PDMS pre-polymer onto the mold for the bottom layer. Place the mold in the desiccator to degas the PDMS for $60 \mathrm{~min}$. Keep the mold overnight in a $60^{\circ} \mathrm{C}$ oven to cure the PDMS. Make sure that it is lying on a horizontal plane.

2. Cool down the PDMS to room temperature. Cut the device to an area of approximately $5.5 \times 5 \mathrm{~cm}^{2}$, which can cover all channel patterns, and peel it off of the mold. Store the fabricated bottom PDMS layer away from ambient dust for later assembly.

4. Fabrication of the PDMS membrane

1. Spin coat approximate $4 \mathrm{~g}$ of PDMS pre-polymer onto a silanized blank wafer at $100 \mathrm{rpm}$ for $90 \mathrm{sec}$ and then $3,000 \mathrm{rpm}$ for 4 sec. Bake the spin-coated wafer in a $60^{\circ} \mathrm{C}$ oven overnight.

5. Assembly of the device

1. Place the fabricated PDMS-PC top layer and the wafer with the spin-coated PDMS membrane in an $\mathrm{O}_{2}$ plasma surface treatment machine with the bonding surfaces facing up. Treat the PDMS surfaces with $90 \mathrm{~W}$ of $\mathrm{O}_{2}$ plasma for $40 \mathrm{sec}$.

2. Bond the top layer onto the PDMS membrane right after the $\mathrm{O}_{2}$ plasma surface treatment. Place a weight (about $600 \mathrm{~g}$ ) on top of the bonded layers and put them in a $60^{\circ} \mathrm{C}$ oven overnight to promote bonding.

3. Cool down the bonded layers to room temperature and cut the membrane bonded to the top layer with a scalpel to an area of approximately $5.5 \times 5 \mathrm{~cm}^{2}$, which can cover all channel patterns. Peel off the bonded structure from the silicon wafer and punch holes at the inlets and outlet of the chemical gradient channel using a $2 \mathrm{~mm}$-diameter biopsy punch.

4. Place the membrane-bonded top layer and the fabricated PDMS bottom layer in the $\mathrm{O}_{2}$ plasma surface treatment machine, with the bonding surfaces facing up, to activate the PDMS surfaces using the plasma at $90 \mathrm{~W}$ for $40 \mathrm{sec}$.

5. Attach the top and bottom layers together for bonding immediately after the surface treatment. Place a weight (about $600 \mathrm{~g}$ ) on top of the entire bonded device and put it in a $60^{\circ} \mathrm{C}$ oven overnight. 
6. Take the entire fabricated device out of the oven and cool it down to room temperature.

\section{Microfluidic Cell Migration Assay}

NOTE: In this paper, we use a commonly used cell line, adenocarcinomic human alveolar basal epithelial cell (A549), and a chemokine, stromal cell derived factor (SDF-1 $\alpha$ ), as examples. For researchers working on other cells and chemokines, please adjust the experimental processes accordingly.

\section{Day 0: Cell preparation}

1. Culture the stock of $A 549$ cells in complete growth medium containing DMEM F12 L-glutamine substitute, $10 \%$ (v/v) fetal bovine serum (FBS), and $1 \%(v / v)$ antimicrob-antimycotic solution. Sub-culture by dissociation with $0.25 \%$ trypsin-EDTA.

2. Prepare cell suspensions for the experiments by centrifuging dissociated cells at $140 \mathrm{xg}$ for 3 min at room temperature. For the microfluidic device experiments, count the cells using a hemocytometer and seed at least $1 \times 10^{6}$ cells in a T75 flask with $10 \mathrm{ml}$ of complete growth medium.

NOTE: All cell culture processes are performed in an incubator with a $37^{\circ} \mathrm{C}$ and $5 \% \mathrm{CO}_{2}$ atmosphere.

\section{Day 1: Device preparation}

1. Place the device into the $\mathrm{O}_{2}$ plasma machine and treat it with $\mathrm{O}_{2}$ plasma at $90 \mathrm{~W}$ for $40 \mathrm{sec}$ to render the microfluidic channel surfaces hydrophilic.

2. Immediately after the surface treatment, install two $14 \mathrm{G}$ blunt needles by directly inserting the needles into the $2 \mathrm{~mm}$ diameter holes punched into the PDMS layer at the chemical gradient channel inlets. Make sure that the needles do not block the microfluidic channels. Draw $0.8 \mathrm{ml}$ of $\mathrm{ddH}_{2} \mathrm{O}$ using a $1 \mathrm{ml}$ syringe with a blunt $14 \mathrm{G}$ needle. Inject the $\mathrm{ddH}_{2} \mathrm{O}$ into the chemical gradient channel from the outlet until the water flows out from both needles at the inlets.

3. Prepare $1 \mathrm{ml}$ of $50 \mu \mathrm{g} / \mathrm{ml}$ fibronectin solution by diluting fibronectin stock with Dulbecco's Phosphate-Buffered Saline (DPBS).

4. Infuse the fibronectin solution from the outlet of the chemical gradient channel using a $1 \mathrm{ml}$ syringe with a blunt $14 \mathrm{G}$ needle until the solution flows out from both needles at the inlets.

5. Incubate the entire device overnight in a conventional cell culture incubator.

\section{Day 2: Cell seeding and microscopy imaging}

1. Cell seeding

1. Aspirate the medium from the cells that were cultured since day 0 and wash the cells with $5 \mathrm{ml}$ of DPBS 2 times. Aspirate the DPBS, add $2 \mathrm{ml}$ of trypsin-EDTA, and incubate the cells in the incubator for $5 \mathrm{~min}$ to detach the cells from the flask surface.

2. Wait until the cells are detached and suspended in the solution and add $8 \mathrm{ml}$ of serum-free medium (DMEM F12 + $1 \%(\mathrm{v} / \mathrm{v})$ antimicrob-antimycotic solution) to the flask. Transfer all of the liquid into a $15 \mathrm{ml}$ conical tube and centrifuge it at $140 \times \mathrm{g}$ for 5 min under room temperature.

3. Aspirate the supernatant after centrifuging and add an appropriate amount of the serum-free medium to make the final cell density $1 \times 10^{6} \mathrm{cells} / \mathrm{ml}$. Take out the microfluidic device prepared on Day 1 . Count the cells using a hemocytometer or another automatic cell counting instrument.

4. Inject serum-free medium from the outlet of the chemical gradient channel using a $1 \mathrm{ml}$ syringe with a blunt $14 \mathrm{G}$ needle until the medium flows out from both needles at the inlets. Inject $200 \mu \mathrm{l}$ of the cell suspension from the outlet of the chemical gradient channel.

5. Observe the cell culture chamber in the device under a microscope to confirm that the cells have been introduced to the microfluidic channel. Place the device into a humid container (e.g., a plastic box with $\mathrm{ddH}_{2} \mathrm{O}$ inside) and keep it in a cell culture incubator for $5 \mathrm{hr}$ to promote the adhesion of the cells onto the device surface.

2. Preparation of reagents for chemical gradient generation

1. Prepare the chemical (SDF-1 $\alpha$ ) at the desired concentration $(100 \mathrm{ng} / \mathrm{ml})$ in serum-free medium. Draw the serum-free medium with and without the chemical into two separate $3 \mathrm{ml}$ syringes connected to high-purity tubing. Set up the syringes on a syringe pump with a flow rate of $1 \mu \mathrm{l} / \mathrm{min}$ for later use.

3. Preparation of the reagents for oxygen gradient generation

1. Make $15 \mathrm{ml}$ of $1 \mathrm{M} \mathrm{NaOH}$ solution and $15 \mathrm{ml}$ of $200 \mathrm{mg} / \mathrm{ml}$ pyrogallol solution. Draw the $\mathrm{NaOH}$ and pyrogallol solutions into two separate $15 \mathrm{ml}$ syringes connected to high-purity PTFE tubing and high-purity tubing, respectively. Set up the syringes on a syringe pump with flow rate of $5 \mu \mathrm{l} / \mathrm{min}$ for later use.

4. Microfluidic device setup

1. After the $5 \mathrm{hr}$ incubation, take the entire device out and place it on a $15 \mathrm{~cm}$ diameter Petri dish. Fix the device in the Petri dish using adhesive putty. Transfer the Petri dish to a live cell imaging microscope in an incubator.

2. Connect the tubing from the syringes for the chemical gradient generation to the inlets of the chemical gradient channel. Connect the outlet to tubing leading to a waste reservoir. Turn on the syringe pump with the syringes for chemical gradient generation.

3. Connect the high-purity tubing from the syringes containing $\mathrm{NaOH}$ and pyrogallol to the inlets of the oxygen gradient channel. Connect tubing to the outlet to collect the waste. Turn on the syringe pump with the syringes for oxygen gradient generation.

4. Add about $15 \mathrm{ml}$ of $\mathrm{ddH}_{2} \mathrm{O}$ to the Petri dish to keep the device moisturized. Set up the live cell imaging microscope for timelapsed image capture, and take an image every $15 \mathrm{~min}$.

\section{Day 3: Data collection and analysis}


1. Transfer the captured image files to a computer. Analyze the images using an open source image analysis software, ImageJ, with an open source plugin for Manual Tracking (https://imagej.nih.gov/ij/plugins/track/track.html) and a free Chemotaxis Tool software (http:// ibidi.com/xtproducts/en/Software-and-Image-Analysis/Manual-Image-Analysis/Chemotaxis-and-Migration-Tool) ${ }^{15,16}$.

\section{Characterization of the Gradients}

NOTE: The chemical and oxygen gradients can be characterized before or after the cell experiments.

1. Numerical simulation of the chemical gradient

1. Estimate the laminar flow nature of microfluidics using computational fluidic dynamics (CFD) simulation.

2. Experimental characterization of the oxygen gradient

1. Prepare an oxygen-sensitive fluorescent dye, tris(2,2'-bipyridyl) ruthenium(III) chloride hexahydrate, solution in water at a concentration of $5 \mathrm{mg} / \mathrm{ml}$.

2. Draw the oxygen-sensitive dye solution into two separate $3 \mathrm{ml}$ syringes connected to high-purity tubing. Set up the syringes on a syringe pump with flow rate of $1 \mu \mathrm{l} / \mathrm{min}$ (identical to the flow rates used for the chemical gradient generation).

3. Connect the tubing from the syringes to the inlets of the chemical gradient channel. Connect the outlet to tubing leading to a waste reservoir. Turn on the syringe pump with the syringes for chemical gradient generation.

4. Measure the fluorescence intensity using an inverted fluorescence microscope with the excitation light passing through a $470 \pm 20 \mathrm{~nm}$ optical filter. Collect the emission light through a $515 \mathrm{~nm}$ long-pass emission filter using a cool CCD camera.

5. Connect the high-purity tubing from the syringes containing $\mathrm{NaOH}$ and pyrogallol to the inlets of the oxygen gradient channel. Connect tubing to the outlet to collect the waste. Turn on the syringe pump with the syringes for oxygen gradient generation.

6. Collect the fluorescence images of the oxygen-sensitive dye flowing in the cell culture chamber.

7. Stop the flow for oxygen gradient generation and disconnect all the tubing to the oxygen generation channel. Connect high-purity tubing from the gas cylinders to the outlet of the oxygen gradient channel.

8. Collect the fluorescence images of the oxygen-sensitive dye flowing in the cell culture chamber when flowing air, pure nitrogen, and pure oxygen into the tubing connected to the oxygen gradient channel.

9. Estimate the oxygen gradients by analyzing the fluorescence images using the Stern-Volmer equation according to References 10 and 11.

\section{Representative Results}

Fabricated PDMS-PC hybrid microfluidic cell culture device. Fig. 1 shows a photo and an illustration of the microfluidic device. The bottom layer contains four levels of serpentine-shaped channels to generate solutions from reagents introduced from two separated inlets with six different mixing ratios. Theoretically, the six different mixing ratios are 1:0, 4:1, 3:2, 2:3, 1:4, and 0:1 (left:right) between the two solutions introduced from the inlets. The chemical gradients constructed by the six different mixing-ratio solutions can be generated in the cell culture chamber, located downstream. The top and bottom layers are separated by a PDMS membrane. In the top layer, the reagents for the oxygen scavenging chemical reaction are introduced into the microfluidic channel from two separate inlets. The reagents are mixed with each other for the reaction immediately before flowing on top of the cell culture chamber to scavenge the oxygen from the bottom channel, without direct chemical contact. The embedded PC film, with a smaller gas diffusion coefficient compared to PDMS, acts as a diffusion barrier that makes oxygen scavenging more efficient. Oxygen gradually diffuses back to the cell culture chamber through the PDMS in the downstream area to form an oxygen gradient along the flow direction. Since the oxygen scavenging chemical reaction is spatially confined, only local oxygen tensions are affected. As a result, the device can be utilized in a conventional cell incubator without altering its global oxygen tension. In the migration experiments, cells are seeded inside the cell culture chamber for observation. The growth medium and chemical reagents are introduced to the device using syringe pumps with precisely controlled flow rates.

Characterization of chemical and oxygen gradients generated inside the device. Due to the laminar flow nature of microfluidics, flow behaviors can be predicted using computational fluidic dynamics (CFD) simulation. In this paper, we constructed a 3D model and performed the simulation using a commercially available multiphysics modeling software. Fig. 2(a) shows a comparison between experimentally characterized fluorescein concentration profiles across the width of the cell culture chamber based on fluorescence intensity measurements and the numerical simulation results. The agreement between the experimental and simulation results suggests that the CFD model can well estimate the chemical gradients generated inside the device. Fig. 2(b) plots the simulated SDF-1a gradient generated in the cell culture chamber. Fig. 3 shows the oxygen gradient characterization results by flowing the oxygen-sensitive fluorescence dye inside the cell culture chamber before the cell experiments. The result indicates that an oxygen gradient, ranging from about 1 to $16 \%$, can be established using the aforementioned protocol. 
Cell migration results. As a demonstration, we performed A549 cell migration studies under 4 combinations of chemokine (SDF-1 $\alpha$ ) and oxygen gradients: (1) no chemokine and no oxygen gradients as a control, (2) with a chemokine gradient and without an oxygen gradient, (3) with an oxygen gradient and without a chemokine gradient, and (4) with both chemokine and oxygen gradients. Fig. 4 shows the photo of the entire experimental setup. The experiments were all performed in a conventional cell culture incubator with the entire setup (including the microfluidic devices, syringe pumps, and live cell imaging microscopes) placed inside it. The cell migration results are shown in Fig. 5. Fig. 5(a) shows the images collected during the experiments using the live cell imaging analyzer, and Fig. 5(b) and (c) plots the cell migration trajectories and average movements under the four combinations analyzed by the ImageJ software with the plugins. The results show that the average cell migration distance in the control approaches zero, which suggests random movement of the cells in the experiment. In contrast, with only the chemokine gradient, the average movement of the cells is towards the left, where the SDF-1a concentration is higher. The results suggest the SDF-1 $\alpha$ chemotaxis behavior of A549 cells, which has been previously reported. In the experiment with only oxygen gradients, the average movement of the cells is upwards, where the oxygen tension is lower. More interestingly, in the experiment with perpendicular chemokine and oxygen gradients, the average movement of the cells is upwards and without any obvious movement in the horizontal direction (chemokine gradient direction).

(a)

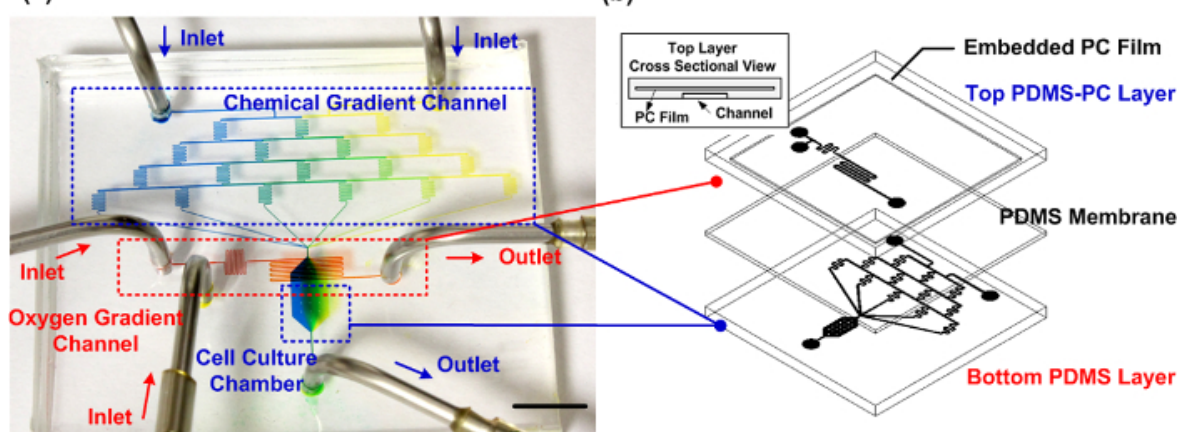

(c) Master Mold for Top Layer

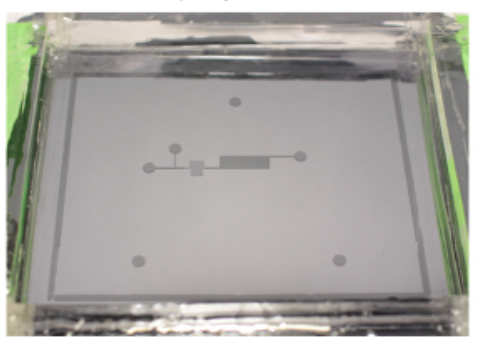

Master Mold for Bottom Layer

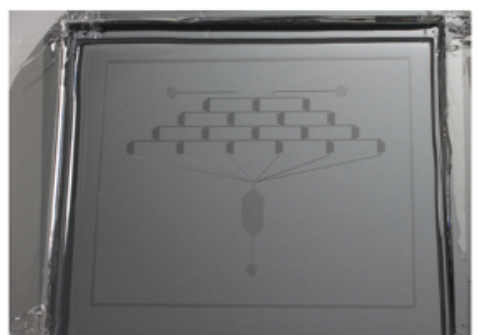

Figure 1: Fabricated PDMS-PC microfluidic cell culture device. (a) The experimental photo of the fabricated device capable of reliably generating perpendicular chemical and oxygen gradients for cell migration studies. The chemical gradient channel is filled with blue and yellow food dyes to demonstrate the gradient generation inside the cell culture chamber. The oxygen gradient channel is filled with red food dye. The scale bar is $1 \mathrm{~cm}$. (b) The schematic of the microfluidic device. The top layer is fabricated using PDMS with an embedded PC layer as a gas diffusion barrier for efficient oxygen gradient control inside the cell culture chamber. (c) The master molds for the fabrication of the top and bottom layers. Please click here to view a larger version of this figure.

(a)

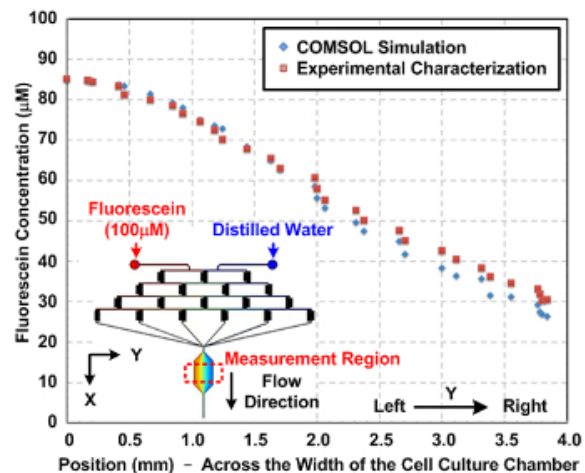

(b)

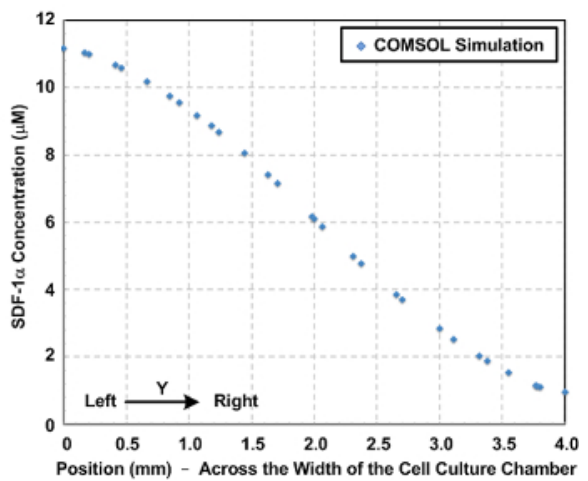

Figure 2: Chemical gradient inside the microfluidic cell culture device. (a) Numerically simulated and experimentally characterized fluorescein concentration gradient inside the cell culture chamber across the width of the cell culture chamber (Y direction). The similarity between the simulated and experimentally measured gradients indicates that the simulation can well predict the chemical gradient. The figure inset shows the three-dimensional (3D) model constructed for the simulation. (b) Numerical simulation result of the SDF-1 $\alpha$ chemokine gradient across the width of the cell culture chamber for cell migration studies. Please click here to view a larger version of this figure. 


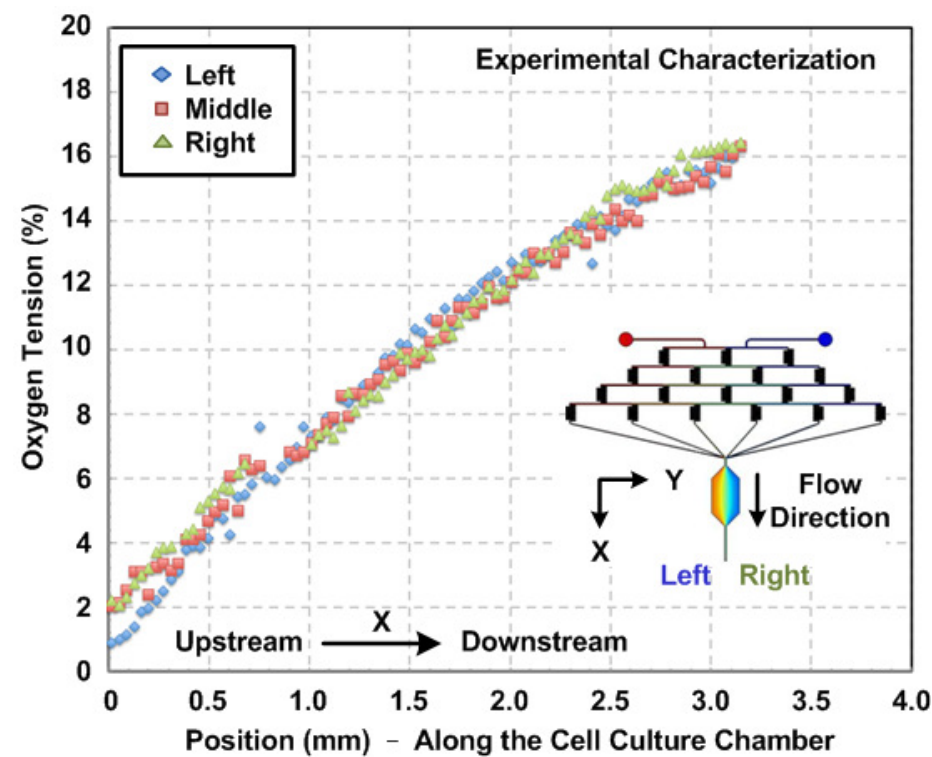

Figure 3: Oxygen gradient inside the microfluidic cell culture device. Experimentally measured oxygen gradients within the cell culture chamber along the flow direction. The gradients were estimated using the oxygen-sensitive fluorescence dye and image analysis. The gradients, from left to right of the chamber, are characterized, and the results show consistent gradient profiles across the width of the chamber.

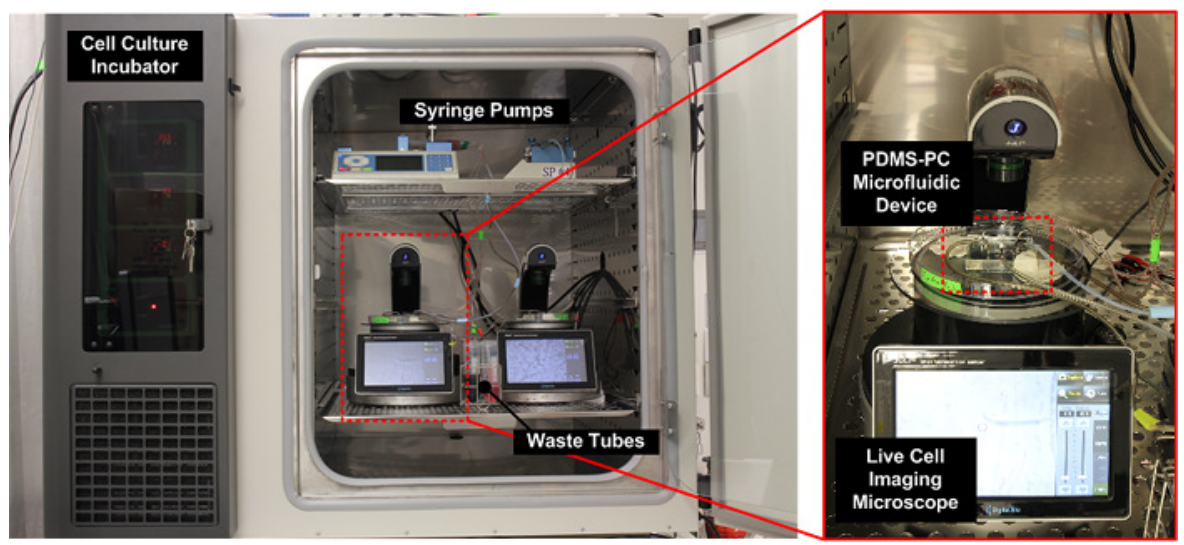

Figure 4: Photos of the experimental setup. The entire setup, including microfluidic devices, syringe pumps, and a live cell imaging microscope, is placed inside a conventional cell culture incubator for optimized cell culture conditions during the experiments. Please click here to view a larger version of this figure. 
(a)
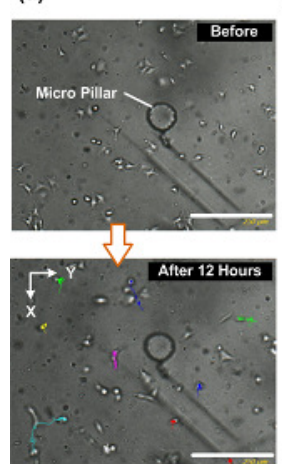

(b)

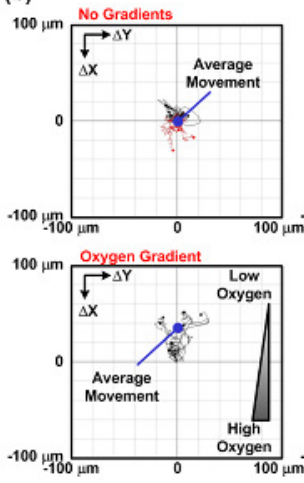

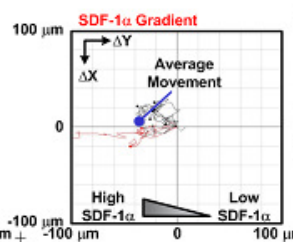

(c)

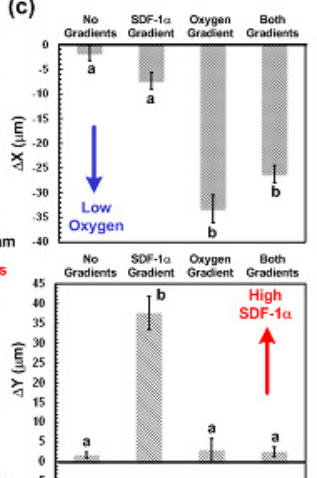

Figure 5: Cell migration study results under perpendicular SDF-1 $\alpha$ and oxygen gradients. (a) Images captured before and after the 12$\mathrm{h}$ cell migration study. The cell migration paths can be analyzed from the captured time-lapsed images using the live cell imaging microscope. (b) The cell migration paths and the analyzed average migration movement from the captured images under 4 different gradient combinations: no gradient, only chemokine gradient, only oxygen gradient, and both chemokine and oxygen gradients. The images were captured every 15 min. The scale bar is $250 \mu \mathrm{m}$. (c) Plots of the average cell migration distances in the perpendicular (oxygen gradient) and horizontal (chemokine gradient) directions under four different gradient combinations. The data are expressed as the mean \pm SD, obtained from three independent experimental sets, and 10 cells were analyzed in each experiment. The statistical significantly different (unpaired Student's t-test, $p<0.01$ ) results are designated by different letters $(a$ and $b)$. Please click here to view a larger version of this figure.

Discussion

The most critical steps to fabricate the PDMS microfluidic device with an embedded PC thin film are: (1) expelling all the bubbles when inserting the PC film into the PDMS pre-polymer while fabricating the PDMS-PC top layer and (2) making sure all the PDMS curing processes are performed on a well-leveled horizontal plane. For cell migration experiments, the most critical steps are: (1) eliminating the bubbles within the microfluidic device, tubing, and syringe pumps during the experiments; (2) ensuring that the microfluidic device is placed on a well-leveled horizontal plane during live cell imaging for the cell migration observation; and (3) keeping the device moisturized by adding dd $\mathrm{H}_{2} \mathrm{O}$ to the $\mathrm{Petri}$ dish during the experiments and making sure that the water is not dried out.

In order to successfully fabricate the PDMS-PC hybrid microfluidic device without delamination, it is critical to remove all bubbles during the PC film insertion. The PC film can be slowly inserted from an angle (about 15 to 30 degrees away from the PDMS pre-polymer surface) to prevent bubbles generation during the insertion of the PC film into the PDMS pre-polymer. If necessary, the entire PDMS pre-polymer with the embedded PC film can be placed in the desiccator connected to the vacuum pump for 10 min to expel the trapped bubbles. If the PC film floats up after the vacuum process, a pipette tip can be used to press the PC film down onto the cured PDMS layer. Repeat the vacuum and press processes if necessary.

For the cell experiments, a lack of air bubbles is critical for the microfluidic cell culture. Make sure that no air bubbles are introduced into the entire microfluidic setup (including syringe pumps, tubing, and the microfluidic device) when making connections. If air bubbles are created within the microfluidic setup due to the decrease of gas solubility under the elevated temperature of the experiments inside the incubator, all the experimental components (including the syringes and tubing) and reagents (including the growth medium, pyrogallol, and $\mathrm{NaOH}$ ) can be placed into the incubator beforehand (at least 20 min prior to usage) to minimize the temperature variation. Syringe pumps often generate heat from the operation of the motors within the pumps. It is usually acceptable to operate syringe pumps inside incubators; however, do check the incubator temperature during the experiments. If the temperature elevates during the experiments, additional cooling procedures need to be carried out. Several feasible cooling methods can be employed, such as placing a box of ice into the incubator, reducing the number of syringe pumps placed inside the incubator, or using an incubator with a force cooling system.

The PDMS-PC microfluidic cell culture device developed in this paper is capable of reliably generating perpendicular chemical and oxygen gradients for cell migration studies. The limitation of the developed device is that the generated oxygen gradient profiles depend on the balance between oxygen flux, driven by chemical reaction scavenging, and oxygen diffusion from the ambient atmosphere, through the device, and into the medium. As a result, the oxygen gradient profiles cannot be arbitrarily controlled inside the device. Compared to existing microfluidic cell culture platforms, the device developed in this paper is the first one capable of performing cell culture studies under combinations of chemical and oxygen gradients. The entire device can be fabricated using the conventional soft lithography replica molding process, without tedious alignment and expensive instrumentation. The gradients can be numerically simulated and experimentally characterized to provide more physiological microenvironment-like conditions for in vitro cell studies. By using a spatially confined chemical reaction method with a PC film as a gas diffusion barrier, the oxygen gradient can be generated without using pressurized gas cylinders and sophisticated gas flow control units. In addition, the setup requires only small amounts of chemicals (less than $10 \mathrm{ml}$ per day) to maintain the oxygen gradients. Since the oxygen tension control is confined locally around the microfluidic channel, and does not disturb the ambient oxygen concentration, the entire setup can be placed inside a conventional cell culture incubator without additional temperature, humidity, and $\mathrm{CO}_{2}$ control instrumentation. As a result, the developed device has great potential to be practically used in biological labs.

Due to technical limitations, cellular behaviors under oxygen tensions have seldom been studied in the existing literature. With the help of the device developed in this paper, cell culture under oxygen gradients can be performed in a facile manner that greatly promotes cell studies under oxygen gradients. Furthermore, a similar operation principle can be applied to generate other physiologically relevant gaseous gradients, such as carbon dioxide and nitric oxide, for in vitro cell culture studies ${ }^{17}$. These capabilities demonstrate that the PDMS-PC microfluidic device shows 
great potential for various cell culture applications, including drug testing and cell proliferation and migration assays, to advance in vitro cell culture studies.

\section{Disclosures}

The authors declare that they have no competing financial interests.

\section{Acknowledgements}

This paper is based on work supported by the National Health Research Institutes (NHRI) in Taiwan under the Innovative Research Grant (IRG) (EX105-10523EI), the Taiwan Ministry of Science and Technology (MOST 103-2221-E-001-001-MY2, 104-2221-E-001-015-MY3, 105-2221E-001-002-MY2), the Academia Sinica Thematic Project (AS-105-TP-A06), and the Research Program in Nanoscience and Nanotechnology. The authors would like to thank Ms. Rachel A. Lucas for proofreading the manuscript.

\section{References}

1. Phillips, J.E., Burns, K.L., Le Doux, J.M., Guldberg, R.E., García, A. J. Engineering graded tissue interfaces. Proc. Natl. Acad. Sci. USA. 105 (34), 12170-12175, 0.1073/pnas.0801988105, (2008).

2. Wang, F. The signaling mechanisms underlying polarity and chemotaxis. Cold Spring Harb. Perspect Biol. 1 (4), 1-16 (2009)

3. Oh, S.H., Ward, C.L., Atala, A., Yoo, J.J., Harrison, B.S. Oxygen generating scaffolds for enhancing engineering tissue survival. Biomaterials. 30 (5), 757-762 (2009).

4. Decaris, M.L., Lee, C.I., Yoder, M.C., Tarantal, A.F., Leach, J.K. Influence of the oxygen microenvironment on the proangiogenic potential of human endothelial colony forming cells. Angiogenesis. 12, 303-311 (2009).

5. Chung, B.G., et al. Human neural stem cell growth and differentiation in a gradient-generating microfluidic device. Lab Chip. 5 (4), 401-406, 10.1039/b417651k, (2005)

6. Harris, A.L. Hypoxia - a key regulatory factor in tumor growth. Nat. Rev. Cancer. 2 (1), 38-47 (2002)

7. Allen, J.W., Bhatia, S.N. Formation of steady-state oxygen gradients in vitro: application to liver zonation. Biotechnol. Bioeng. 82 (3), $253-262$, 10.1002/bit.10569, (2003).

8. McCord, A.M., Jamal, M., Shankavaram, U.T., Lang, F.F., Camphausen K., Tofilon P.J. Physiologic oxygen concentration enhances the stemlike properties of CD133+ human glioblastoma cells in vitro. Mol. Cancer Res. 7 (4), 489-497 (2009).

9. Lo, J.F., Sinkala, E., Eddington, D.T., Oxygen gradients for oprn well cellular cultures via microfluidic substrates. Lab Chip. 10 (18), 2394-2401 (2010).

10. Chang, C.-W. et al. A polydimethylsiloxane-polycarbonate hybrid microfluidic device capable of generating perpendicular chemical and oxygen gradients for cell culture studies. Lab Chip. 14 (19), 3762-3772 (2014).

11. Chen, Y.-A., et al. Generation of oxygen gradients in microfluidic devices for cell culture using spatially confined chemical reactions. Lab Chip. 11 (21), 3626-3633 (2011).

12. Peng, C.-C., Liao, W.-H., Chen, Y.-H., Wu, C.-Y., Tung, Y.-C. A microfluidic cell culture array with various oxygen tensions. Lab Chip. 13 (16), 3239-3245 (2013).

13. Xia, Y. and Whitesides, G. M. Soft Lithography, Ann. Rev. Mate. Sci. 28, 153-184 (1998)

14. Friend, J., Yeo, L. Fabrication of microfluidic devices using polydimethylsiloxane. Biomicrofluidics. 4 (2), 026502 (2010).

15. Cordelières, F. P. Manual Tracking. Institute Curie, Orsay, France, https://imagej.nih.gov/ij/plugins/track/Manual\%20Tracking\%20plugin.pdf, (2005).

16. Asano, Y., Horn, E. Instructions Chemotaxis and Migration Tool 2.0. ibidi GmbH, Martinsried, Germany, Germany, http://ibidi.com/fileadmin/ products/software/chemotaxis_tool/IN_XXXXX_CT_Tool_2_0.pdf?x14743=a3676cb19a79449e2b05d5ecb4a8aae6, (2013).

17. Chen, Y.-H., Peng, C.-C., Cheng, Y.-J., Wu, J.-G., Tung, Y.-C. Generation of nitric oxide gradients in microfluidic devices for cell culture using spatially controlled chemical reactions. Biomicrofluidics. 7 (6), 064104 (2013). 